\title{
Subsynchronous resonance oscillations mitigation via fuzzy controlled novel braking resistor model
}

\author{
M. Fayez ${ }^{1}$, M. Mandour ${ }^{2}$, M. El-Hadidy ${ }^{3}$, F. Bendary ${ }^{4}$ \\ 1,2,4Electrical Engineering Department, University of Benha, Egypt \\ ${ }^{3}$ Egyptian Electricity Holding Company (EEHC), Egypt
}

\begin{tabular}{l} 
Article Info \\
\hline Article history: \\
Received Nov 9, 2018 \\
Revised Apr 1, 2019 \\
Accepted May 2, 2019 \\
\hline Keywords: \\
Chopper rectifier controlled \\
braking resistor \\
Fuzzy logic control \\
Series compensation \\
Subsynchronous \\
resonance (SSR) \\
Thyristor controlled \\
braking resistor
\end{tabular}

\begin{abstract}
Subsynchronous resonance (SSR) torsional torque oscillations is a problem of a great concern in the power engineering community. SSR causes torsional torque oscillations with ever-increasing magnitudes occurring in the machine shaft sections causing a premature fatigue life expenditure of the shaft metal. In this paper, dynamic braking switching strategy designed through fuzzy logic control theory and implemented via novel braking resistor model, namely chopper rectifier controlled braking resistor for tempering SSR torsional torque oscillations of a large turbo-generator. The proposed mitigation scheme has been tested on the IEEE second benchmark model for SSR studies. Comparative simulation study via MATLAB/Simulink-based modeling and simulation environment of the test model with and without the suggested mitigation regime should demonstrate its effectuality for mitigation of SSR torsional torque oscillations.
\end{abstract}

Copyright (C) 2019 Institute of Advanced Engineering and Science. All rights reserved.

\section{Corresponding Author:}

Mohamed Fayez, Electrical Engineering Department, University of Benha, Shoubra, Cairo 108, Egypt.

Email: eng_mf69@yahoo.com

\section{INTRODUCTION}

Usually, the rotor of a steam turbine-generator set is considered as a single rotational mass with certain inertia constant in the analysis and investigation of many power system dynamic problems [1]. But, the rotor of a steam turbine-generator is a highly complicated mechanical structure. The rotor of a steam turbinegenerator set (i.e. shaft train) is composed of a string of dominant massive elements, each one has a considerable inertia constant and relatively large diameter, rigidly connected in tandem at the thinner shaft extensions [1]. The complete shaft train can surpass 50 meters of total length and weight several hundred tons [1]. The spring-mass model of the turbine-generator shaft is prerequisite for elucidating the torsional torque oscillations occurring at the shaft extensions [1]. SSR torsional torque oscillations is a problem of a great concern in the power engineering community $[1,2]$. SSR is a transmission system-based problem that causes adverse devastating effects on the turbine-generator sets operating under this circumstance [2]. Therefore, SSR is considered as multidisciplinary power utility problem.

In 1970, and again in 1971, a 909 MVA cross-compound turbine-generator at Mohave coal-fired power plant in southern Nevada, California, USA, had encountered collector ring damage in the high-pressure unit exciter shaft due to SSR torsional torque oscillations [2, 3]. The devastating consequences of Mohave accidents have fed off the industry's enthusiasm about this phenomenon to find reliable mitigation countermeasures and underscored the urge for quickly implementing them [3]. SSR causes torsional torque oscillations with ever-increasing magnitudes occurring in the machine shaft sections causing a premature fatigue life expenditure of the shaft metal [1-5]. The shaft is then definitely destined to experience irrevocable 
low-cycle fatigue cracks or even a complete shaft fracture [1-5]. SSR arises in power plants, especially with steam-turbine prime movers, as an undesirable collateral result for transmission lines emanating from a power plant and equipped with series capacitors at certain conditions [1-5]. The fatigue damage of large turbinegenerator shaft is definitely undesirable and catastrophic event in the power industry $[5,6]$. This is because the shaft rehabilitation process is very expensive and takes several months to be accomplished during which the unit is intuitively out of service [6]. Also, during the shutdown shaft rehabilitation period, the plant should purchase the electric energy required to energize the auxiliary equipments of the plant from the utility which brings more economic casualties to the plant owner beside the inability to sell the output electrical energy of the plant [6].

Series compensation has been widely utilized by the utility since 1952 for economically transmitting bulk power transactions over long transmission distances [7]. Series compensation has many overwhelming benefits. It aggrandizes the electric power transferring capability of long transmission corridor, enhances the voltage profile of the grid, and boosts the angular stability of the system and so forth [7]. At certain cases, series compensated power systems are vulnerable to have SSR condition in which the series compensated electric system interchanges a considerable amount of energy with a turbine-generator mechanical system at one more of the natural frequencies of the combined electromechanical system below the synchronous frequency of the electric system [4-6].

For the sake of secure operation of bulk power systems, large turbo-generators are certainly needed to be in service for as long as possible due to their active and reactive power supplying capabilities [5]. So, utilizing protection relays to protect large units from SSR conditions will not generally be considered except as a final sanctuary [5]. Therefore, SSR mitigation countermeasures should alleviate the devastating SSR torque oscillations and eliminate the need to trip the units that are prone to resonant condition. There have been a considerable number of technical studies and research papers addressing the SSR problem and presenting many mitigation countermeasures with control algorithms, mitigation equipment sizes, and the most effective location in the network for these prospective mitigation candidates [6].

Several mitigation approaches have been developed for tempering of SSR oscillations such as, Static VAR Compensator (SVC), Thyristor-Controlled Series Capacitor (TCSC), Static Synchronous Series Compensator (SSSC), Static Compensator (STATCOM), Unified Power Flow Controller (UPFC), and excitation system control through using an appropriate supplementary stabilizing signal [8-10].

Among the multiple presently conceivable SSR mitigation candidates, the dynamic braking resistor (BR) is an extremely effective and relatively cheap mitigation candidate for SSR problems [10-15]. BR was initially implemented for augmenting transient stability of electric power systems [1]. BR functions as a pseudo electric load with the ability to consume the momentarily accelerating energy in power systems arising due to severe nearby faults, especially in power systems with hydro dominated power plants [1]. Therefore, by dissipating the extra generated power, BR should prohibit generator pole slipping conditions and thereby augmenting the transient stability of electric power systems [1]. Bonneville Power Administration (BPA) dynamic brake is one of the largest existing BR installations in realistic power systems with power dissipation ability of $1400 \mathrm{MW}$ with $230 \mathrm{kV}$ rated voltage [1]. Many works of literature have addressed the utilization of $\mathrm{BR}$ connected to the grid via a three-phase $\mathrm{AC}$ voltage controller, i.e. thyristor controlled braking resistor (TCBR), for mitigation of multi-modal shaft torsional oscillations [15-20]. TCBR per phase model is constructed as two back-to-back connected high-power thyristor valves with BR in series which means that three BRs were utilized for SSR torsional oscillations mitigation [16-20].

Very recently, a novel BR model, namely Chopper Rectifier Controlled Braking Resistor (CRCBR) model, is introduced to the academic community as an alternative innovative mean for implementing the concept of dynamic braking interventions for enhancing the transient stability of power systems [21-23]. In $[21,22]$, the effectiveness of CRCBR for transient stability enhancement in single machine infinite bus (SMIB) system and the Western System Coordinated Council (WSCC), 3-machine 9-bus system, respectively, have been tested. CRCBR controlled through a fuzzy logic controller (FLC) is proposed in this article for mitigation of SSR torsional oscillations. CRCBR is fundamentally a single BR bank linked to the three-phase terminals of the synchronous generator through a three-phase full-wave uncontrolled rectifier diode bridge and chopper switch (CS) [21, 22].

No similar work could be found in the literature to date regarding the utilization of CRCBR for mitigation of SSR torsional torque oscillations. The CRCBR is either switched ON or switched OFF based on the energization signal produced by the FLC. The generator mass speed deviation is implemented in this work as a local control input signal to the FLC for strategizing the CRCBR switching intervals. The influence of the proposed scheme in mitigating SSR torsional oscillations is tested in this work using the IEEE second benchmark model via MATLAB/Simulink-based modeling and simulation environment. Comparative simulation study of the test system with and without the suggested mitigation regime should demonstrate its effectuality for mitigation of SSR torsional torque oscillations. 
The rest of this paper is organized as follows. In section 2, the well-known test system is briefly described. In section 3 the concept of utilizing the FLC to orchestrate the switching strategy of the CRCBR is introduced. In section 4, MATLAB/Simulink time domain simulation results are delineated with comments. In section 5, the key conclusions of this work are drawn. Finally, the references used in this work are listed.

\section{SYSTEM MODEL}

To scrutinize the effectiveness of the suggested mitigation regime in this paper, the well-known IEEE second benchmark model for computer simulation of SSR is adopted. Figure 1 depicts the single line diagram of the system under study together with CRCBR and the steam turbine-generator shaft detail [21-23]. The IEEE second benchmark model is basically a single synchronous generator $(600 \mathrm{MVA} / 22 \mathrm{kV} / 60 \mathrm{~Hz} / 3600 \mathrm{rpm})$ connected to an infinite bus through generator step-up transformer $(600 \mathrm{MVA} / 22 \mathrm{kV} / 500 \mathrm{kV})$ and two extrahigh voltage (EHV) transmission lines, one of which is equipped with a series capacitor of $55 \%$ seriescompensation ratio. The generator is driven by one single-flow high-pressure (HP) steam turbine and one double-flow low-pressure (LP) steam turbine connected in tandem. The model of CRCBR consists of one BR bank connected to the generator terminals via six-pulse full wave uncontrolled Rectifier Bridge and a chopper switch as shown in Figure 1 [21-23]. In this article, the Insulated Gate Bipolar Transistor (IGBT) is utilized as a chopper switch. The BR bank is assumed to be capable of dissipating $50 \%$ the connected generator apparent power (i.e. $300 \mathrm{MW}$ ).

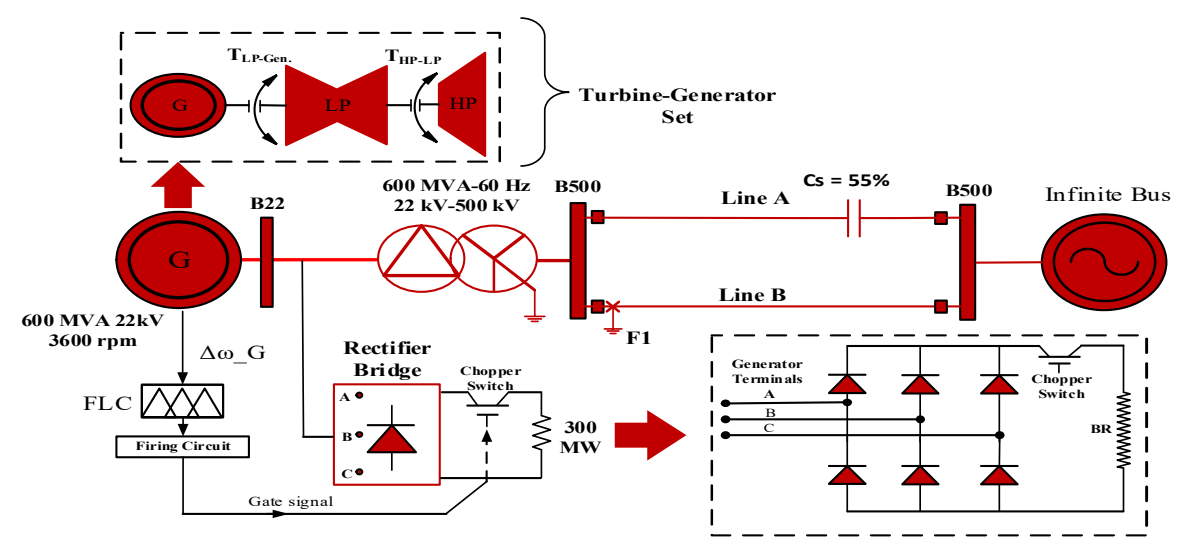

Figure 1. The IEEE second benchmark model with a turbine generator shaft multi-mass model incorporating chopper rectifier controlled braking resistor device [21-23]

The brake is switched ON or OFF via the chopper switch to mitigate the shaft torsional oscillations by reducing the net available energy for acceleration and hence speed deviation of the generator mass due to severe disturbances such as system close in faults [1]. The proposed scheme utilizes a local control signal represented by the generator mass speed deviation to help the FLC determine whether the CRCBR should be switched ON or switched OFF. The system is completely modeled in MATLAB/Simulink environment. The electrical parameters for the synchronous generator are obtained from [23]. The under-study steam turbine configuration is appropriately illustrated in [23]. It consists of HP section and single LP section. The turbine contributing torque fractions for the turbine sections HP, and LP are 50\%, and 50\%, respectively [23]. The detailed turbine-generator shaft mechanical data is obtained from [23]. The electrical parameters of the system in per-unit, with 100 MVA as a common base power, are also obtained from [23].

\section{FUZZY LOGIC CONTRLLER DESIGN}

The fuzzy logic is differing from the crisp logic in Boolean theory which utilizes only two logical levels ( 0 or 1 ) in that it uses unbounded logical levels (from 0 to 1 ) to deal with issues that have uncertainties or obscure situations [24-26]. FLCs are demonstrated to be more robust and their performances possess a lesser sensitivity to the parametric differences than the traditional controllers [24-26]. Recently, fuzzy-logic control has gained a reputation in effectively solving many power system dynamic problems [24-26]. FLC is a nonlinear controller with a rule-based system, in other words, it is an artificial-intelligence (AI) based system 
which relied on the use of expert knowledge [24-26]. This expert knowledge is commonly acquired through conducting comprehensive mathematical modeling, analysis, and development of control algorithms for the power systems [24-26]. A set of fuzzy linguistic rules, usually distinguished by "IF-THEN" statements, representing a control decision-making mechanism responsible for regularizing the effectuations for system stimulants [24-26]. The basic FLC configuration consists of the following four prime stages: fuzzification, knowledge base, inference engine, and defuzzification [24-26]. In the fuzzification stage, the crisp input values are mapped into fuzzy variables by using normalized membership functions and occasionally input weighting factors [24-26]. The fuzzy-logic inference engine appropriately deduces the suitable control action based on the attainable rule base [24]. Two major inference engine types are Takagi-Sugeno (TS), and Mamdani [27]. The fuzzy control action is converted to proper crisp value through the defuzzification process using normalized membership functions and occasionally output weighting factors [24-26].

TS type is more efficient than the Mamdani type for dynamic systems with fast changing dynamics because it works well with linear, adaptive and optimization techniques and it is well suited to mathematical analysis [27]. Therefore, TS inference mechanism is utilized in the simulation study. The overlapping of the membership functions makes the mapping smoother and enhances the controller performance [24].

It has proven that the damping level obtained from using the generator mass speed deviation as the controller input is better than the damping level obtained from using other turbine sections speed deviation signals $[17,20]$. Also, the generator mass speed signal is not so difficult to capture like HP, and LP speed signals since the entire steam turbine is rigorously sealed with the outer cases via steam seal system. So, the generator mass speed deviation in per-unit, $\Delta \omega$, is selected as input to the FLC while the output signal is constant with either 0 or 1 . The output signal of FLC is then sent to the IGBT gating circuit which produces the gate signals for the chopper switch based on the controller decision output.

The Gaussian and Sigmoid inputs membership functions are shown in Figure 2 in which three linguistic variables, NB (Negative Big), Zo (Zero) and PB (Positive Big), are defining the fuzziness of the controller input. The membership functions parameters are determined by trial and error according to the generator mass speed deviation swing range based on an extensive simulation study. The output type is constant having either 0 or 1 values ( 0 for both $\mathrm{ZO}$ and NB, 1 for PO). Where 0 indicates that the IGBT should be in OFF state and 1 indicates that the IGBT should be in ON state.

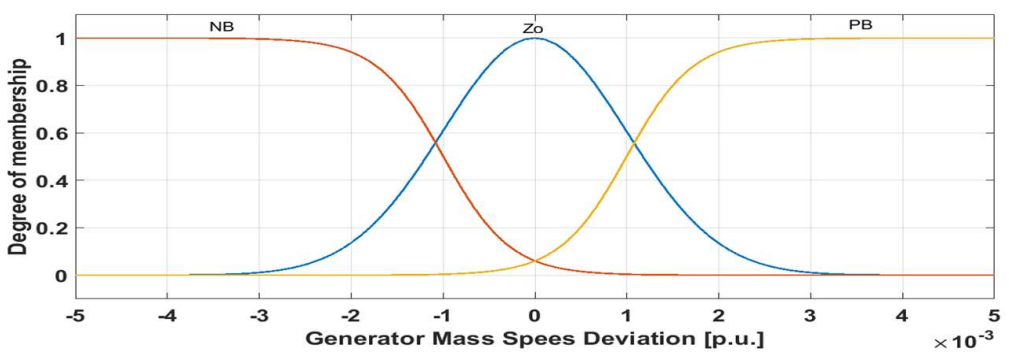

Figure 2. FLC input membership functions in per-unit

The proposed control scheme is very straightforward and simple since it has only three control rules where the BR is inserted if the generator speed deviation exceeds a certain value (the acceleration state) and removed elsewhere (steady state and deceleration state). There are three premise membership functions depicted in Figure 2, one for each rule, and the conclusions are singleton so the fuzzy control rules are as follows, If the input $(\Delta \omega)$ is NB then the output is 0 , If the input $(\Delta \omega)$ is Zo then the output is 0 and If the input $(\Delta \omega)$ is PB then the output is 1 .

\section{SIMULATION RESULTS}

To further demonstrate the effectiveness of the proposed mitigation regime, time domain simulation study via Simulink model is carried out. Three-phase to ground (3LG) self-healing fault is applied at line B, very close to generator high voltage bus at fault point F1, as shown in Figure 1. The fault is applied at 0.25 second from the simulation time of 5 seconds. The fault is self-cleared after 0.0169 seconds from the inception of the disturbance. The torsional torque profiles of turbine-generator shaft system in per-unit (p.u.) and the speed responses of turbine-generator different masses with and without the fuzzy-controlled CRCBR are listed as a family of curves shown in Figure 3 and Figure 4. 


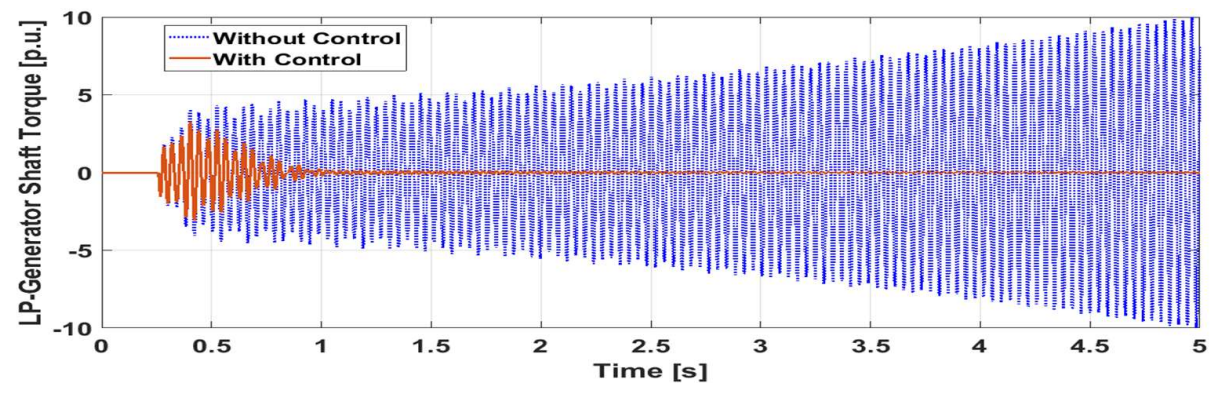

(a)

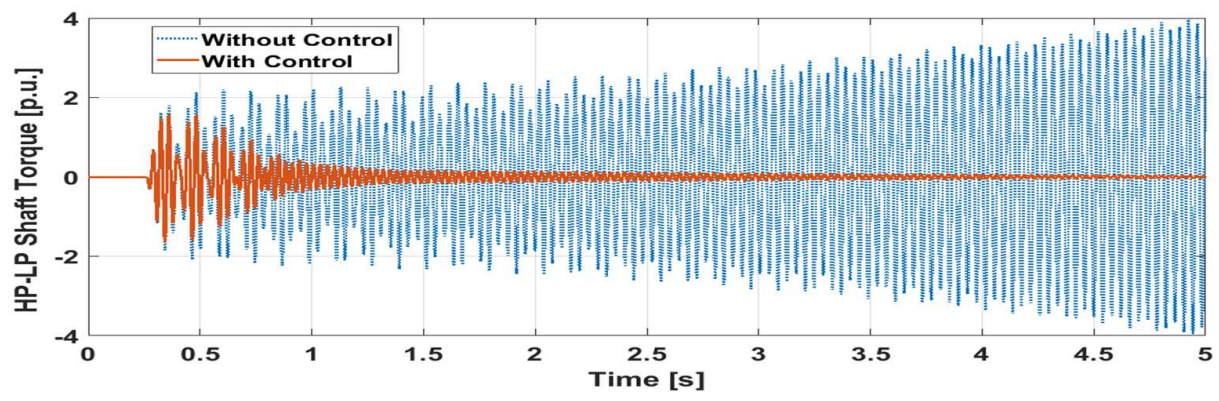

(b)

Figure 3. Torque responses with and without fuzzy controlled CRCBR due to the three-phase self-healing fault at the generator HV bus in p.u., (a) LP turbine-generator shaft torsional torque, (b) HP-LP Shaft torsional torque

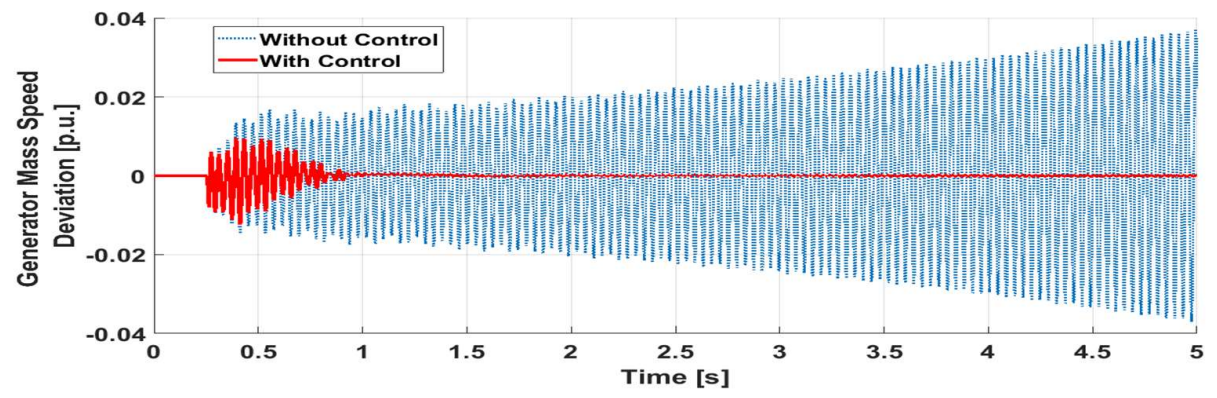

(a)

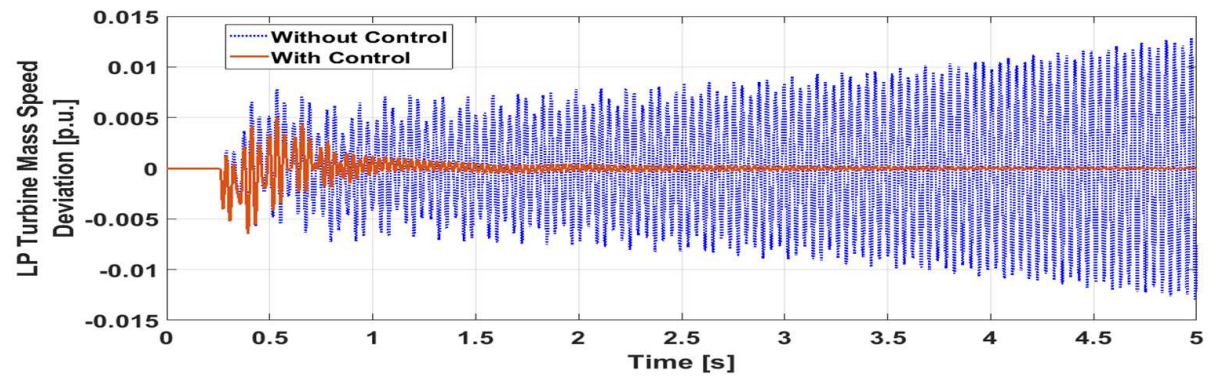

(b) 


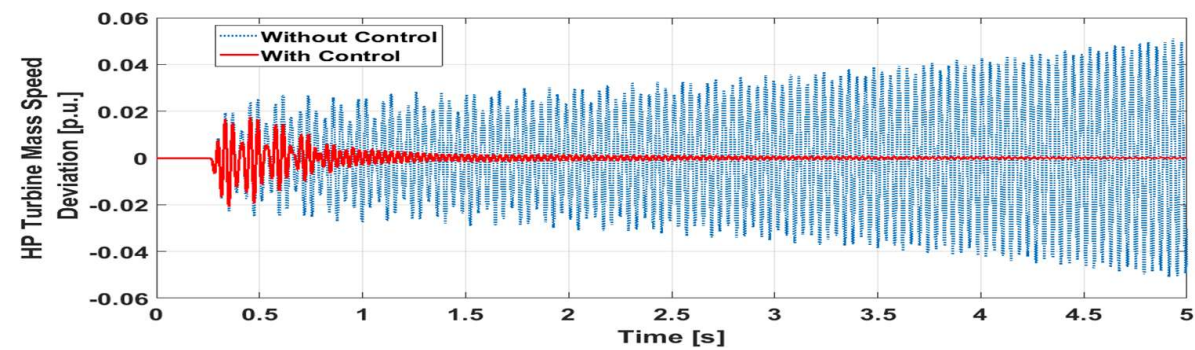

(c)

Figure 4. Relative turbine-generator mass speed responses due to the three-phase self-healing fault at the generator HV bus in p.u., (a) Generator mass speed deviation, (b) LP turbine mass speed deviation,

(c) HP turbine mass speed deviation.

Finally, in Figure 5 it is shown the comparative responses of the mechanical rotor angle deviation for the synchronous generator under the given fault condition. Due to applying the proposed technique the oscillations in the rotor angle is settled after 1 second from the perturbation initiation.

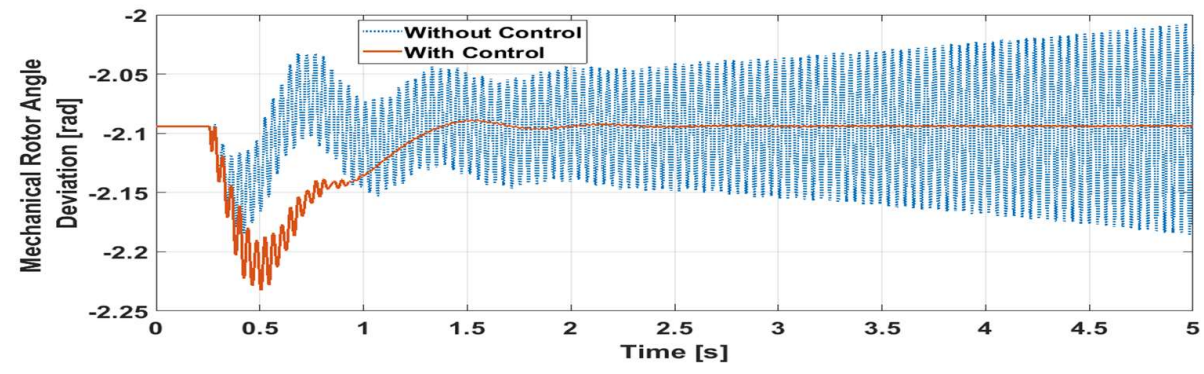

Figure 5. Mechanical rotor angle deviation in [rad]

As evidenced from the simulation results portrayed in Figure 3, Figure 4 and Figure 5, in the base case simulation results without the proposed scheme, it is clearly seen that both speed and torque responses seem to be sustained with ever-increasing amplitudes in the time frame of the simulation which indicates the unstable nature of the responses. The driving mechanical torque transmitted between the shaft's masses have experienced devastating oscillations with extremely unacceptable amplitudes. These torque oscillations will, for sure, end up causing a premature shaft fatigue life expenditure and irrevocable shaft cracks or even a shaft fracture with the unpleasant outcomes. Due to applying the fuzzy controlled CRCBR, it is obviously shown that the shaft torsional oscillations of a turbine-generator set and the speed responses have experienced an excellent supplementary damping, which further enables the utilizing of series compensation safely near the steam power plants without any mechanical jeopardy.

\section{CONCLUSION}

This paper authenticates the effectiveness of CRCBR controlled via FLC to damp SSR shaft torsional oscillations. From the simulation results, the speed and the torque profiles of the machine manifest a significantly good supplementary damping which allows the torsional oscillations and the speed oscillations to die out quickly. Utilizing only one BR unit, instead of three units in case of TCBR, might encourage the utilities to use it for damping the shaft torsional oscillations arising from SSR conditions and compromise between the benefits of implementing series compensation and the concerns of the machine shaft damage. The CRCBR is considered as a viable mean for boosting the security of the power system from the operational perspective by extending the fatigue life of the units in the power system to their maximum possible potential by neutralizing any SSR condition. Finally, implementing the proposed scheme should capacitate conventional series compensation on transmission lines emanating from thermal power plants safely and soundly without jeopardizing the shaft mechanical integrity of the turbine-generator set. 


\section{REFERENCES}

[1] M. Fayez Ahmed, et al., "Torsional Oscillations Mitigation for Interconnected Power System via Novel Fuzzy Control Based Braking Resistor Model," Conseil International des Grands Réseaux Électriques (CIGRÉ), Paris, France, August 26-31, pp. 1-9, 2018

[2] EPRI Technical Report 1011679, "Steam Turbine-Generator Torsional Vibration Interaction with The Electrical Network," Electric Power Research Institute (EPRI), 2005.

[3] Geoff Klempner and Isidor Kerszenbaum, Handbook of Large Turbo-Generator Operation and Maintenance. John Wiley \& Sons, 2008.

[4] Duncan N. Walker, Torsional Vibration of Turbomachinery. McGraw-Hill, 2003.

[5] P. M. Anderson, Power system protection. John Wiley \& Sons, 1999.

[6] Leonard L. Grigsby, Power system stability and control, Third edition. CRC Press, 2012.

[7] Mircea Eremia, Chen-Ching Liu, and Abdel-Aty Edris, Advanced solutions in power systems HVDC, FACTS and artificial intelligence. John Wiley \& Sons, 2016.

[8] R. Mohan Mathur and Rajiv K. Varma, Thyristor-based FACTS controllers for electrical transmission systems. John Wiley \& Sons, 2002.

[9] Shruthi Ramachandra, et al., "Dynamic bifurcation analysis and mitigation of SSR in SMIB system," Indonesian Journal of Electrical Engineering and Computer Science, vol. 15, no. 3, 2019.

[10] S. Helmy, et al., "Mitigating subsynchronous resonance torques using dynamic braking resistor," International Middle East Power Systems Conference (MEPCON), Egypt, 2010, pp. 416-421.

[11] Li Wang, and Ching-Huei Lee, "Application of dynamic resistance braking on stabilizing torsional oscillations," in International Conference on Computers, Communications and Automation, Beijing, China, 1993, pp. 145-148.

[12] R. M. Hamouda, et al., "Damping Torsional Oscillations in Large Turbo-Generators Using Thyristor Controlled Braking Resistors," in 2008 Australasian Universities Power Engineering Conference (AUPEC'08), Australasia, 2008, pp. 1-6.

[13] A. H. Rahim and H. M. Al-Maghraby, "Dynamic Braking Resistor for Control of Subsynchronous Resonant Modes," IEEE Power Engineering Society Summer Meeting, Seattle, WA, USA, 2000, pp. 1930-1935.

[14] A. H. M. A. Rahim and H. M. AI-Maghraby, "Control of SSR Oscillations Through A Quasi-Optimum Dynamic Braking Strategy," International Journal of Power and Energy, vol. 23, pp. 90-95, 2003.

[15] A. H. M. A. Rahim, "A Minimum-Time Based Fuzzy Logic Dynamic Braking Resistor Control for Sub-Synchronous Resonance," Electrical Power and Energy Systems, vol. 26, pp. 191-198, 2004.

[16] O. Wasynczuk, "Damping Shaft Torsional Oscillations Using A Dynamically Controlled Resistor Bank," IEEE Transactions on Power Apparatus and Systems, vol. 100, pp. 3340-3349, 1981.

[17] EPRI Technical Report 103902, "Dynamic Brake Control to Reduce Turbine Shaft Transient Torque," Electric Power Research Institute (EPRI), 1994.

[18] M. H. Ali, et al., "Minimization of Shaft Torsional Oscillations by Fuzzy Controlled Braking Resistor Considering Communication Delay," WSEAS Transactions on Power Systems, vol. 3, pp. 174-179, 2008.

[19] M. H. Ali, et al., "Coordination of Fuzzy Controlled Braking Resistor and Optimal Reclosing for Damping ShaftTorsional Oscillations of Synchronous Generator," in International Conference on Electrical Machines and Systems (ICEMS), Seoul, South Korea, 2007, pp. 1259-1264.

[20] M. H. Ali, et al., "A fuzzy Logic Controlled Braking Resistor Scheme for Damping Shaft Torsional Oscillations," IEEJ Transactions on Power and Energy, vol. 124, pp. 207-214, 2004.

[21] R. Saluja and M. H. Ali, "Novel Braking Resistor Models for Transient Stability Enhancement in Power Grid System," IEEE PES Innovative Smart Grid Technologies (ISGT) Conference, Washington DC, USA, 2013, pp. 1-6.

[22] R. Saluja, S. Ghosh, and M. H. Ali, "Transient stability enhancement of multi-machine power system by novel braking resistor models," Proceedings of the IEEE Southeast Conference, Florida, USA, 2013, pp. 1 - 6.

[23] IEEE Committee Report, "Second Benchmark Model for Computer Simulation of Subsynchronous Resonance," IEEE Transactions on Power Apparatus and Systems, vol. 104, pp. 1057-1066, 1985.

[24] M. Fayez Ahmed, et al., "Torsional Oscillations Mitigation via Novel Fuzzy Control Based Braking Resistor Model," International Electrical Engineering Journal (IEEJ), vol. 7, pp. 2173-2181, 2016.

[25] Gilfred Allen Madrigal, et al., "Fuzzy logic-based maximum power point tracking solar battery charge controller with backup stand-by AC generator," Indonesian Journal of Electrical Engineering and Computer Science, vol. 16, no. 1, pp. 136-146, 2019.

[26] Salam Waley Jeaeb, et al., “Advanced optimal by PSO-PI for DC motor," Indonesian Journal of Electrical Engineering and Computer Science, vol. 16, no, 1, pp. 165-175, 2019.

[27] S. Mohagheghi, et al., "Modified Takagi-Sugeno Fuzzy Logic-Based Controllers for A Static Compensator in A Multi-Machine Power System," in Industry Applications Conference, Seattle, USA, 2004, pp. 2637-2642.

Int. J. of Adv. in Appl. Sci. Vol. 8, No. 2, June 2019: 164 - 170 